\title{
Spin decoherence due to Fluctuating Fields
}

\author{
Piotr Szańkowski, ${ }^{1,2}$ M. Trippenbach, ${ }^{1}$ and Y. B. Band ${ }^{2}$ \\ ${ }^{1}$ Institute of Theoretical Physics, University of Warsaw, ul. Hoża 69, PL-00-681 Warszawa, Poland \\ ${ }^{2}$ Department of Chemistry, Department of Physics and Department of Electro-Optics, \\ and the Ilse Katz Center for Nano-Science, Ben-Gurion University, Beer-Sheva 84105, Israel \\ The dynamics of a spin in the presence of a deterministic and a fluctuating magnetic field is \\ solved for analytically to obtain the averaged value of the spin as a function of time for various \\ kinds of fluctuations (noise). Specifically, analytic results are obtained for the time dependence of \\ the expectation value of the spin, averaged over fluctuations, for Gaussian white noise, Guassian \\ colored noise, as well as non-Gaussian telegraph noise. Fluctuations cause the decay of the average \\ spin vector (decoherence). For noise with finite temporal correlation time, a deterministic component \\ of the field can suppress decoherence of the spin component along the field. Hence, decoherence can \\ be manipulated by controlling the deterministic magnetic field. A simple universal physical picture \\ emerges which explains the mechanism for the suppression of decay.
}




\section{INTRODUCTION}

Quantum systems are almost invariably coupled to an environment that degrades their coherence. Decoherence of quantum systems is a fundamental problem, with implications across all branches of physics. The temporal evolution of a spin system in the presence of coupling to an environment is perhaps the simplest phenomenon involving decoherence. For example, a particle of spin 1/2, which can be mapped onto any two-level system (TLS) [1], and vice versa, has served as an important paradigm [2 5]. Specific examples include the decoherence of a qubit in a quantum-computer or quantum-information processor [6], spin dynamics in a magnetometer 7], accuracy and stability limitations of atomic clocks due to interactions with an environment [8], decoherence of two-level quantum dot systems [9, 10], nitrogen vacancy centers in diamond [11, 12].

Under certain assumptions, which we discuss in the next section, the evolution of a spin in the presence of an environment can be represented by evolving the spin in an effective magnetic field, $\vec{B}^{(\text {eff })}=\vec{B}_{0}+\vec{B}_{E}(t)$. Here $\vec{B}_{0}$ is a deterministic magnetic field (which can be time-dependent) that can be used to exert control over the spin. The magnetic field $\vec{B}_{E}(t)$ models the influence of an environment on the system, and is represented by a vector stochastic process $\vec{b}(t)$. Averaging over fluctuations corresponds to tracing out the environmental degrees of freedom. This yields a reduced, non-unitary dynamics wherein the averaged spin decoheres in time.

The physical properties of the environment determine the statistical properties of $\vec{B}_{E}(t)$, which in turn determine the type of stochastic process $\vec{b}(t)$. Here we consider several types of stochastic fluctuations. A prototype model for fluctuations is Gaussian white noise [13, 14], a random process having vanishing correlation time. As a consequence, the system driven by it evolves without memory, since it has no way to probe the past. A natural generalization is a Gaussian colored noise [15, 16], wherein the random process has a finite correlation time, hence, the system evolves with memory. The ubiquitousness of this type of noise can be attributed to central limit theorem which states that the superposition of a very large collection of independent sources of fluctuation tends to Gaussian noise. Moreover, in some spin systems there are more than one source of noise which differ in their intensity and correlation time scale. For example, in the nitrogen-vacancy diamond system [17], NV centers experience slowly fluctuating noise from ${ }^{13} \mathrm{C}$ impurity nuclear magnetic moments, and extremely fast fluctuations from nearby electronic nitrogen spins. The former can be modeled by colored noise, which is characterized by a very long correlation time, while the latter can be treated as a white noise, since it sets the fastest time scale in the system. A particular case of colored noise that is not Gaussian, telegraph noise, has been studied in connection with quantum dot qubit systems [4, 5]. Reference [3] claims that telegraph noise can be used to model the environment of systems where a spin can interact with relatively few random fluctuators in its neighborhood, and these fluctuators go back and forth between only two states.

The idea of using stochastic methods for modeling an environment or other complex interactions within the spin system is not new. For example, the problem of the line shape in paramagnetic resonance is discussed in Ref. [18]. It was assumed that the electrons in an atom are affected by a perturbation which varies randomly in time at a rate determined by exchange interactions. As a result, the fluctuations around the stationary state are induced and their effect on the absorption spectra can be calculated. Another example is presented in Ref. [19], where the authors consider an ensemble of spins interacting with an environment as well as each other. The problem of calculating the effects of these complex interactions on the observed line shape is circumvented by introducing stochastic fluctuations of the precession frequency of the observed spins.

The goal of this work is to develop methods to describe and analyze the process of relaxation of a single spin induced by contact with an environment. An important result of our analysis is that, when the spin evolves with memory, it can be manipulated by applying a sufficiently strong deterministic magnetic field $\vec{B}_{0}$ to significantly suppress decoherence. We hope that the level of generality and overall simplicity of our approach allows for a straightforward application to appropriate experimental systems.

The outline of the this paper is as follows. Section II develops the equations of motion for a spin in the presence of a deterministic magnetic field and a stochastically fluctuating magnetic field. Section III describes evolution of the system with Gaussian colored noise fluctuations. In that section we present a simple, intuitive view of the nature of the decay of the averaged spin due to field fluctuations. We also show that this decay can be suppressed when the deterministic magnetic field is strong enough, and the correlation time is long enough (i.e., when the system has memory). Section IV develops the white noise limit of colored noise. Section V explains the effects of memory on decoherence, and Sec. VI considers telegraph noise, which is a specific from of non-Gaussian noise. Finally, a summary and a conclusion are presented in Sec. VII. 


\section{SPIN DYNAMICS IN A FLUCTUATING MAGNETIC FIELD}

We consider fluctuations in an otherwise deterministic system that results from the application of a effective random field generated by an environment with which it interacts. The back-coupling of the spin to the source of the noise is not taken into account (Van Kampen [13] calls this external noise). Hence, the stochastic properties of the noise result only from the environment which is unaffected by the system. We assume that these properties can be measured or otherwise deduced. For each particular system, one should carefully check whether the back-action can indeed be neglected. Examples of systems for which back-action was neglected include: nitrogen-vacancy centers in diamond affected by magnetic moments of impurity spins [12, 20], electron spin in a quantum dot affected by nuclear magnetic moments that fluctuate due to crystal lattice vibrations [10, 21, and magnetic noise in atom chips caused by fluctuations of electron currents in wires that make up the atom chip 22].

Consider a particle of spin $\vec{S}$ with magnetic moment $\vec{\mu}=\mu \vec{S}$ in the presence of a time-dependent field $\vec{B}(t)$. The dynamics is determined using a Zeeman-type Hamiltonian,

$$
H=-\vec{\mu} \cdot \vec{B}(t)=\vec{\Omega}(t) \cdot \vec{S},
$$

where $\vec{S}$ is the vector of spin operators satisfying commutation relations $\left[S_{i}, S_{j}\right]=i \hbar \epsilon_{i j k} S_{k}, \vec{\Omega}(t)=-\mu \vec{B}(t) / \hbar$ is the Rabi frequency vector, $\vec{B}(t)$ is a total magnetic field felt by the particle, $\mu=g \mu_{0}$ is the magnetic moment of the particle, $g$ is the $g$-factor and $\mu_{0}$ is the Bohr (or nuclear) magneton. The magnetic field $\vec{B}(t)$ is the sum of a deterministic field, $\vec{B}_{0}$, whose direction defines the $z$-axis, and a fluctuating field, $\vec{b}(t)$. Hence, the Rabi frequency is given by

$$
\vec{\Omega}(t)=\left(\begin{array}{c}
0 \\
0 \\
\Omega_{0}(t)
\end{array}\right)+\left(\begin{array}{c}
\omega_{x}(t) \\
\omega_{y}(t) \\
\omega_{z}(t)
\end{array}\right)
$$

where $\vec{\Omega}_{0}=-\mu \vec{B}_{0} / \hbar$ and $\vec{\omega}(t)=-\mu \vec{b}(t) / \hbar$. For simplicity, we have taken the deterministic field to have a component only along the $z$-axis. If the deterministic field varies slowly in time (adiabatically), our conclusions below remain valid, but for deterministic fields that vary more quickly, the simple version of the deterministic part of the evolution matrix that satisfies Eq. (7) requires revision, as detailed below.

In the Heisenberg representation, the evolution of the spin operators is given by the equations,

$$
\frac{d}{d t} S_{i}^{(H)}(t)=\frac{\imath}{\hbar}\left[H(t), S_{i}^{(H)}(t)\right]
$$

where the superscript $(H)$ denotes the Heisenberg picture. In our case, the Hamiltonian contains a stochastic term, $\vec{\omega}(t)$ :

$$
H(t)=\Omega_{0}(t) S_{z}+\vec{\omega}(t) \cdot \vec{S}
$$

The commutator on the right hand side of the Heisenberg equation of motion, Eq. (3), is easy to evaluate, and leads to the vector form of the equation,

$$
\frac{d}{d t} \vec{S}^{(H)}(t)=\left\{\Omega_{0}(t) \vec{e}_{z}+\vec{\omega}(t)\right\} \times \vec{S}^{(H)}(t)
$$

and the initial condition is simply

$$
\vec{S}^{(H)}(0)=\left(\begin{array}{c}
S_{x} \\
S_{y} \\
S_{z}
\end{array}\right)
$$

The solution to Eq. (5) with the initial condition (6) can be written as a $3 \times 3$ evolution matrix, $\hat{U}(t, 0)$, acting on the initial vector, $\vec{S}^{(H)}(0)$. It is convenient to write the equations, $S_{i}^{(H)}(t)=\sum_{j=1}^{3} U_{i j}(t, 0) S_{j}^{(H)}(0)$, in vector notation as $\vec{S}^{(H)}(t)=\hat{U}(t, 0) \vec{S}^{(H)}(0)$, where the hat ${ }^{\wedge}$ indicates a $3 \times 3$ matrix. For convenience, let us take the deterministic field $\Omega_{0}$ to be constant in time. Substituting into the equations of motion yields,

$$
\frac{d}{d t} \hat{U}(t, 0)=\left(\Omega_{0} \hat{\varepsilon}^{z}+\sum_{k=1}^{3} \omega_{k}(t) \hat{\varepsilon}^{k}\right) \hat{U}(t, 0) .
$$


Here the matrix elements of $\hat{\varepsilon}^{k}$ are defined as $\varepsilon_{i j}^{k} \equiv \epsilon_{i k j}$, where $\epsilon_{i k j}$ is the Levi-Civita symbol. The matrices $\hat{\varepsilon}^{i}$ satisfy commutation relations similar to those of angular momentum operators,

$$
\left[\hat{\varepsilon}^{i}, \hat{\varepsilon}^{j}\right]=\epsilon_{i j k} \hat{\varepsilon}^{k} .
$$

Consequently, they are generators of rotations in a real, three-dimensional vector space. For example, $\hat{\varepsilon}^{z}$ generates rotations around $z$ axis,

$$
\hat{R}(\theta, z) \equiv \exp \left\{\theta \hat{\varepsilon}^{z}\right\}=\left(\begin{array}{ccc}
\cos \theta & -\sin \theta & 0 \\
\sin \theta & \cos \theta & 0 \\
0 & 0 & 1
\end{array}\right) .
$$

The evolution operator $\hat{U}$ is a rotation operator that causes precession of the spin vector around the instantaneous rotation axis defined by the total field $\vec{\Omega}(t)=\vec{\Omega}_{0}+\vec{\omega}(t)$. The equation of motion for the spin does not depend upon the details of the representation of the spin, hence the solution to the dynamics for an arbitrary spin is also given by (77). The only indication of the spin representation, i.e., the dimension $2 S+1$ of the representation, is in the initial condition.

Experimental measurements of the spin will of necessity correspond to quantum expectation values and averages over the stochastic fluctuations of the magnetic field. Hence the quantity $\langle\overrightarrow{\vec{S}(t)}\rangle$ corresponds to experimental measurements of the spin. Here, the symbol \langle\rangle means quantum average, and the symbol ${ }^{-}$means average over stochastic variables. Note that the average over fluctuations and the average over the initial quantum state factorizes in the following way: $\langle\overrightarrow{\vec{S}(t)}\rangle=\overline{\hat{U}(t, 0)}\langle\vec{S}(0)\rangle$.

For spin $1 / 2$ (i.e., for a TLS), the average expectation value of the spin $\vec{S}=\hbar \vec{\sigma} / 2$ fully determines the state of the system. This is because the density matrix $\varrho$ can be expressed using the Pauli matrices $\vec{\sigma}$ which, together with identity operator 1 , form a basis in the space of $2 \times 2$ hermitian matrices. In the Schrödinger representation,

$$
\overline{\varrho(t)}=\frac{1}{2}\left(1+\operatorname{Tr}\left\{\overline{\vec{\sigma}^{(H)}(t)} \varrho(0)\right\} \cdot \vec{\sigma}\right)=\frac{1}{2}(1+\overline{\langle\vec{\sigma}(t)\rangle} \cdot \vec{\sigma}) .
$$

and the expectation values of the spin at time $t$ is given by vector parameter $\vec{\lambda}(t) \equiv\langle\overline{\vec{\sigma}(t)}\rangle$. Thus, at any time $t$, there are three parameters, $\lambda_{i}(t), i=x, y, z$, which completely specify the density matrix. This does not mean that the dynamics of the density matrix for any $(2 S+1)$-level system can be represented in terms of the dynamics of a particle with spin $\mathbf{S}$ coupled to a magnetic field, since the number of parameters necessary to specify the density matrix larger than the three components of the spin [23].

\section{GAUSSIAN COLORED NOISE}

First, let us consider a process that is Gaussian, isotropic and Markovian [13]. The field fluctuates in all three dimensions with independent components (isotropy) and statistical properties of each component is completely determined by the first two moments (Gaussian), the average and the correlation function. The stationary condition means that the correlation function depends on the time difference and the average is time independent. According to Doob's theorem [24], if the process is also Markovian, the correlation function is exponential and the average is zero, i.e.,

$$
\begin{aligned}
\overline{\omega_{i}(t)} & =0, \\
\overline{\omega_{i}(t) \omega_{j}\left(t^{\prime}\right)} & =\kappa\left(t-t^{\prime}\right) \delta_{i j}=\omega_{0}^{2} e^{-\frac{\left|t-t^{\prime}\right|}{\tau_{c}}} \delta_{i j} .
\end{aligned}
$$

Here the bar indicates average over the fluctuations, $\kappa\left(t-t^{\prime}\right)$ is the correlation function with correlation time $\tau_{c}$, the average fluctuating Rabi frequency vanishes, $\overline{\vec{\omega}}=0$, and the variance of the Rabi frequency, $\omega_{0}^{2}$, specifies the strength of the fluctuations.

Several comments regarding Guassian colored noise are in order. First, note that the Gaussian process can model a wide variety of environments due to the central limit theorem; if the environment consists of many individual and independent fluctuating elements, the central limit theorem substantiates the approximation of using a Gaussian process as the effective field affecting the spin. Secondly, if there is no back-action of the spin on the environment, and the environment is in a stationary state (e.g., is in equilibrium) then the process is stationary as well. Finally, the isotropy assumption regarding the random magnetic field produced by the environment is appropriate if no particular special direction can be associated with the environment. 
To solve the equation of motion (7), first we eliminate the so called drift term due to the deterministic part of the magnetic field, $\Omega_{0} \hat{\varepsilon}^{z} \hat{U}(t, 0)$, from the right hand side of the equation by transforming to the rotating frame. Then Eq. (7) takes the form

$$
\frac{d}{d t} \hat{U}^{\prime}(t, 0)=\left(\vec{\omega}(t) \cdot \overrightarrow{\hat{\varepsilon}}^{\prime}(t)\right) \hat{U}^{\prime}(t, 0) .
$$

In this equation we introduced transformed matrices, denoted by a prime, $\overrightarrow{\hat{\varepsilon}}^{\prime}(t)$ and $\hat{U}^{\prime}(t, 0)$, which are given by

$$
\begin{aligned}
\left(\varepsilon^{\prime}\right)_{i j}^{k}(t) & \equiv \sum_{m, n} R_{i m}\left(t \Omega_{0}, z\right) \varepsilon_{m n}^{k} R_{n j}\left(-t \Omega_{0}, z\right)=R_{k l}\left(t \Omega_{0}, z\right) \varepsilon_{i j}^{l} \\
U_{i j}^{\prime}\left(t_{1}, t_{2}\right) & \equiv \sum_{m, n} R_{i m}\left(t_{1} \Omega_{0}, z\right) U_{m n}\left(t_{1}, t_{2}\right) R_{n j}\left(-t_{2} \Omega_{0}, z\right)
\end{aligned}
$$

where $\hat{R}\left(t \Omega_{0}, z\right)=\exp \left\{t \Omega_{0} \hat{\varepsilon}^{z}\right\}$ is a deterministic Euclidean rotation matrix that results in counterclockwise rotation around the $z$-axis by the angle $\Omega_{0} t$ [see Eq. (9)]. Note that the scalar product $\vec{\omega}(t) \cdot \overrightarrow{\hat{\varepsilon}^{\prime}}(t)$ in Eq. (13) can be rewritten as

$$
\vec{\omega}(t) \cdot \overrightarrow{\hat{\varepsilon}}^{\prime}(t)=\left(\hat{R}\left(-t \Omega_{0}, z\right) \vec{\omega}(t)\right) \cdot \overrightarrow{\hat{\varepsilon}} \equiv \vec{\omega}^{\prime}(t) \cdot \overrightarrow{\hat{\varepsilon}}
$$

The time-dependent rotation matrix $\hat{R}\left(t \Omega_{0}, z\right)$ "mixes" field fluctuations in the $x$ and $y$ component. In the Lab frame, $\omega_{x}$ and $\omega_{y}$ are independent, however, because of the mixing, they are correlated in the rotating frame and the correlation functions are modified in the following way:

$$
\begin{aligned}
& \overline{\omega_{x}^{\prime}\left(t_{1}\right) \omega_{y}^{\prime}\left(t_{2}\right)}=-\overline{\omega_{y}^{\prime}\left(t_{1}\right) \omega_{x}^{\prime}\left(t_{2}\right)}=\kappa\left(t_{1}-t_{2}\right) \sin \left[\Omega_{0}\left(t_{1}-t_{2}\right)\right], \\
& \overline{\omega_{x}^{\prime}\left(t_{1}\right) \omega_{x}^{\prime}\left(t_{2}\right)}=\overline{\omega_{y}^{\prime}\left(t_{1}\right) \omega_{y}^{\prime}\left(t_{2}\right)}=\kappa\left(t_{1}-t_{2}\right) \cos \left[\Omega_{0}\left(t_{1}-t_{2}\right)\right], \\
& \overline{\omega_{z}^{\prime}\left(t_{1}\right) \omega_{i}^{\prime}\left(t_{2}\right)}=\kappa\left(t_{1}-t_{2}\right) \delta_{3 i} .
\end{aligned}
$$

The stochastic field in the rotating frame, $\vec{\omega}^{\prime}(t)$, remains a Gaussian process, since it is a linear combination of Gaussian processes. Note that any time-independent rotation does not change the correlation functions of the isotropic Gaussian vector process $\vec{\omega}(t)$.

The formal solution to Eq. (13) is given in the terms of the time-ordered exponential function,

$$
\hat{U}^{\prime}(t, 0)=\mathcal{T} \exp \left\{\sum_{i} \hat{\varepsilon}^{i} \int_{0}^{t} \omega_{i}^{\prime}(\tau) d \tau\right\},
$$

defined by the power series, $\mathcal{T} \exp \int_{0}^{t} \hat{A}(\tau) d \tau \equiv \hat{1}+\int_{0}^{t} d \tau_{1} \hat{A}\left(\tau_{1}\right)+\int_{0}^{t} d \tau_{1} \int_{0}^{\tau_{1}} d \tau_{2} \hat{A}\left(\tau_{1}\right) \hat{A}\left(\tau_{2}\right)+\ldots$, where $\mathcal{T}$ is the timeordering operator [25]. The average value of $\hat{U}^{\prime}, \overline{\hat{U}^{\prime}(t, 0)}$, can be computed by noting that it has the form of a moment generating functional $\left\{M_{X}[\xi(t)]\right\}$ for matrix processes, $\hat{X}_{i}(t) \equiv \omega_{i}^{\prime}(t) \hat{\varepsilon}^{i}, i=x, y, z$, where $\xi(t)=\left(\xi_{x}(t), \xi_{y}(t), \xi_{z}(t)\right)$ is the vector of trial functions [? ]. Then, the average can be "absorbed" inside the exponential function by expressing $M_{X}[\xi(t)]$ through a cumulant generating functional $\left\{K_{X}[\xi(t)]\right\}$ in terms of cumulant averages denoted here by a double bar (see Ref. [26] for a discussion of the cumulant expansion methods used here):

$$
\begin{aligned}
& \overline{\hat{U}^{\prime}(t, 0)}=\overline{\mathcal{T} \exp \left\{\sum_{i} \hat{\varepsilon}^{i} \int_{0}^{t} \omega_{i}^{\prime}(\tau) d \tau\right\}}=\left.M_{X}[\xi(t)]\right|_{\substack{\xi(t) \equiv 1 \\
\hat{X}_{i} \equiv \omega_{i}^{\prime} \hat{\varepsilon}^{i}}} \equiv \\
& \equiv \mathcal{T} \exp \left\{K_{\omega \hat{\varepsilon}}[1]\right\}=\mathcal{T} \exp \left\{\overline{\mathcal{T} \exp \left(\sum_{i} \hat{\varepsilon}^{i} \int_{0}^{t} \omega_{i}^{\prime}(\tau) d \tau\right)-\hat{1}}\right\}= \\
& =\mathcal{T} \exp \left\{\sum_{i, j} \hat{\varepsilon}^{i} \hat{\varepsilon}^{j} \int_{0}^{t} d \tau_{1} \int_{0}^{\tau_{1}} d \tau_{2} \overline{\overline{\omega_{i}^{\prime}\left(\tau_{1}\right) \omega_{j}^{\prime}\left(\tau_{2}\right)}}\right\} .
\end{aligned}
$$

In the last step, we took advantage of the fact that all cumulants beyond the second vanish for Gaussian processes, and $\overline{\overline{\omega_{i}^{\prime}(t) \omega_{j}^{\prime}\left(t^{\prime}\right)}}=\overline{\omega_{i}^{\prime}(t) \omega_{j}^{\prime}\left(t^{\prime}\right)}$ if the average is zero. 
Substituting the correlation functions given by Eqs. (17), (18) and (19), we find the following expession for the cumulant generating functional $K$ evaluated at $\xi(t) \equiv 1$,

$$
K_{\omega^{\prime} \hat{\varepsilon}}[1]=\left(\hat{\varepsilon}^{z}\right)^{2} \int_{0}^{t} d \tau \gamma(\tau)+\left(\left(\hat{\varepsilon}^{x}\right)^{2}+\left(\hat{\varepsilon}^{y}\right)^{2}\right) \int_{0}^{t} d \tau \gamma_{+}(\tau)+\left(\hat{\varepsilon}^{x} \hat{\varepsilon}^{y}-\hat{\varepsilon}^{y} \hat{\varepsilon}^{x}\right) \int_{0}^{t} d \tau \gamma_{-}(\tau),
$$

where the rates $\gamma$ are given by integrals of the correlation functions:

$$
\begin{aligned}
\gamma(t) & =\int_{0}^{t} d \tau \overline{\omega_{z}^{\prime}(\tau) \omega_{z}^{\prime}(0)}=\int_{0}^{t} d \tau \kappa(\tau), \\
\gamma_{+}(t) & =\int_{0}^{t} d \tau \overline{\omega_{x}^{\prime}(\tau) \omega_{x}^{\prime}(0)}=\int_{0}^{t} d \tau \overline{\omega_{y}^{\prime}(\tau) \omega_{y}^{\prime}(0)}=\int_{0}^{t} d \tau \kappa(\tau) \cos \left(\Omega_{0} \tau\right), \\
\gamma_{-}(t) & =\int_{0}^{t} d \tau \overline{\omega_{x}^{\prime}(\tau) \omega_{y}^{\prime}(0)}=-\int_{0}^{t} d \tau \overline{\omega_{y}^{\prime}(\tau) \omega_{x}^{\prime}(0)}=\int_{0}^{t} d \tau \kappa(\tau) \sin \left(\Omega_{0} \tau\right) .
\end{aligned}
$$

$K$ can be further simplified if we take into account properties of the rotation generators $\hat{\varepsilon}^{i}$. From the commutation relations (8), the commutator in the third term of Eq. (22) is equal to $\hat{\varepsilon}^{z}$. It is easy to verify that the sum of squares of $\hat{\varepsilon}^{x}$ and $\hat{\varepsilon}^{y}$ in the second term of $K$ can be written as $\hat{\varepsilon}^{x 2}+\hat{\varepsilon}^{y 2}=-\left(2 \hat{1}+\hat{\varepsilon}^{2}\right)$, hence, the parts of Eq. (22) commute with each other. Thus, the average evolution matrix, which is equal to the exponential of $K$, factorizes into three parts,

$$
\begin{aligned}
\overline{\hat{U}^{\prime}(t, 0)} & =\mathcal{T} \exp \left\{K_{\omega^{\prime} \hat{\varepsilon}}[1]\right\}=\exp \left\{\hat{\varepsilon}^{z 2} \int_{0}^{t} d \tau \gamma(\tau)-\left(2 \hat{1}+\hat{\varepsilon}^{z 2}\right) \int_{0}^{t} d \tau \gamma_{+}(\tau)+\hat{\varepsilon}^{z} \int_{0}^{t} d \tau \gamma_{-}(\tau)\right\}= \\
& =\underbrace{\left(\begin{array}{ccc}
e^{-\Gamma(t)} & 0 & 0 \\
0 & e^{-\Gamma(t)} & 0 \\
0 & 0 & 1
\end{array}\right)}_{\begin{array}{c}
\text { decay caused by } \\
\text { the fluctuations in } z
\end{array}} \underbrace{\left(\begin{array}{ccc}
e^{-\Gamma_{+}(t)} & 0 & 0 \\
0 & e^{-\Gamma_{+}(t)} & 0 \\
0 & 0 & e^{-2 \Gamma_{+}(t)}
\end{array}\right)}_{\begin{array}{c}
\text { decay caused by } \\
\text { the fluctuations in } x y \text { plane }
\end{array}} \underbrace{\left(\begin{array}{ccc}
\cos \Gamma_{-}(t) & -\sin \Gamma_{-}(t) & 0 \\
\sin \Gamma_{-}(t) & \cos \Gamma_{-}(t) & 0 \\
0 & 0 & 1
\end{array}\right)}_{\begin{array}{c}
\text { precession frequency modification } \\
\text { due to fluctuations in } x y \text { plane }
\end{array}},
\end{aligned}
$$

where

$$
\begin{aligned}
\Gamma_{+}(t) & =\int_{0}^{t} d \tau \gamma_{+}(\tau)=\left(\frac{\omega_{0}^{2} \tau_{c}^{2}}{1+\Omega_{0}^{2} \tau_{c}^{2}}\right)\left[\frac{t}{\tau_{c}}+\left(\frac{1-\Omega_{0}^{2} \tau_{c}^{2}}{1+\Omega_{0}^{2} \tau_{c}^{2}}\right)\left(e^{-\frac{t}{\tau_{c}}} \cos \Omega_{0} t-1\right)-\left(\frac{2 \Omega_{0} \tau_{c}}{1+\Omega_{0}^{2} \tau_{c}^{2}}\right) e^{-\frac{t}{\tau_{c}}} \sin \Omega_{0} t\right] \\
\Gamma_{-}(t) & =\int_{0}^{t} d \tau \gamma_{-}(\tau)=\left(\frac{\omega_{0}^{2} \tau_{c}^{2}}{1+\Omega_{0}^{2} \tau_{c}^{2}}\right)\left[\Omega_{0} t+\left(\frac{1-\Omega_{0}^{2} \tau_{c}^{2}}{1+\Omega_{0}^{2} \tau_{c}^{2}}\right) e^{-\frac{t}{\tau_{c}}} \sin \Omega_{0} t+\left(\frac{2 \Omega_{0} \tau_{c}}{1+\Omega_{0}^{2} \tau_{c}^{2}}\right)\left(e^{-\frac{t}{\tau_{c}}} \cos \Omega_{0} t-1\right)\right], \\
\Gamma(t) & =\int_{0}^{t} d \tau \gamma(\tau)=\omega_{0}^{2} \tau_{c}\left[t-\tau_{c}\left(1-e^{-t / \tau_{c}}\right)\right] .
\end{aligned}
$$

The first term on the right hand side of Eq. (26) describes the decay caused by the fluctuations in the direction parallel to the applied field $\vec{\Omega}_{0}=\Omega_{0} \hat{e}_{z}$. These fluctuations affect only spin components perpendicular to the $z$ axis, and the decay rate $\Gamma(t)$ does not depend on the intensity of the constant field. The second term originates from the fluctuations in the $x y$ plane, which is perpendicular to the applied field. Fluctuations in the plane couple to all the components of the spin and cause their decay. The key feature is that the rate of decay due to these fluctuations is dependent on $\Omega_{0}$. Finally, the third term, also related to the fluctuations in the $x y$ plane, describes the modification of the precession frequency of the system caused by the noise.

The averaged expectation values of the spin are given by:

$$
\begin{aligned}
\left\langle\overline{S_{x}(t)}\right\rangle & =e^{-\left[\Gamma(t)+\Gamma_{+}(t)\right]}\left\{\cos \left[\Omega_{0} t+\Gamma_{-}(t)\right]\left\langle S_{x}\right\rangle-\sin \left[\Omega_{0} t+\Gamma_{-}(t)\right]\left\langle S_{y}\right\rangle\right\}, \\
\left\langle\overline{S_{y}(t)}\right\rangle & =e^{-\left[\Gamma(t)+\Gamma_{+}(t)\right]}\left\{\sin \left[\Omega_{0} t+\Gamma_{-}(t)\right]\left\langle S_{x}\right\rangle+\cos \left[\Omega_{0} t+\Gamma_{-}(t)\right]\left\langle S_{y}\right\rangle\right\}, \\
\left\langle\overline{S_{z}(t)}\right\rangle & =e^{-2 \Gamma_{+}(t)}\left\langle S_{z}\right\rangle .
\end{aligned}
$$

In the limit $\Omega_{0} \rightarrow 0$, we obtain $\Gamma_{+}(t) \rightarrow \Gamma(t)$ and $\Gamma_{-}(t) \rightarrow 0$, hence Eqs. (30)-(32) reduce to

$$
\langle\overline{\vec{S}(t)}\rangle \underset{\Omega_{0} \rightarrow 0}{\longrightarrow} e^{-2 \Gamma(t)}\langle\vec{S}\rangle .
$$

Substituting Eq. (29) into (33) yields the analytic form (33) of the isotropic decay of the average spin, regardless of the initial state of the spin, as can be seen in Fig. 1 For times $t \gg \tau_{c}$, the decay is simply exponential with the rate 


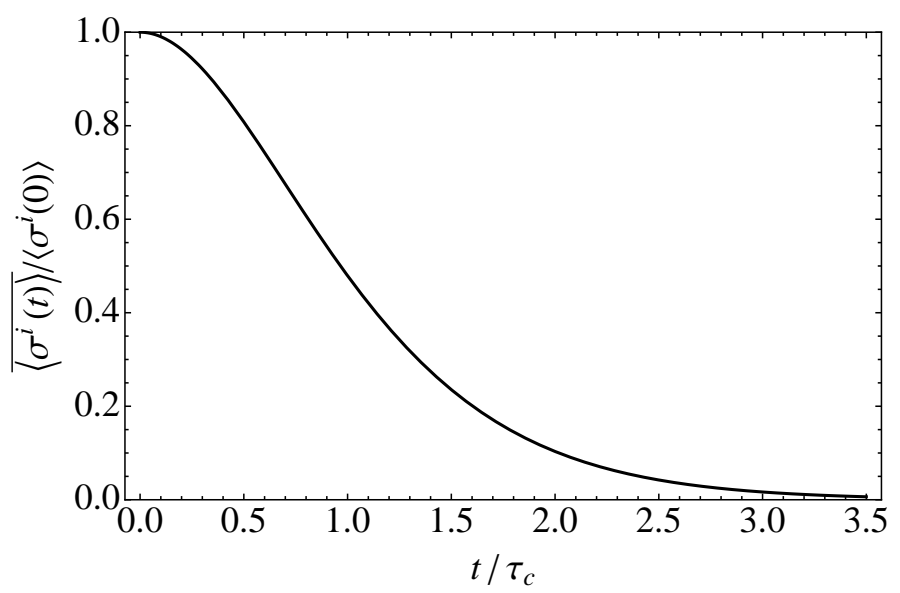

FIG. 1. The expectation value of the spin, $\langle\overline{\vec{S}(t)}\rangle$, subject to Gaussian colored noise, versus time for $\Omega_{0}=0$ and $\omega_{0} \tau_{c}=1$. The analytic formula for $\langle\overline{\vec{S}(t)}\rangle$ is given in Eq. (33).

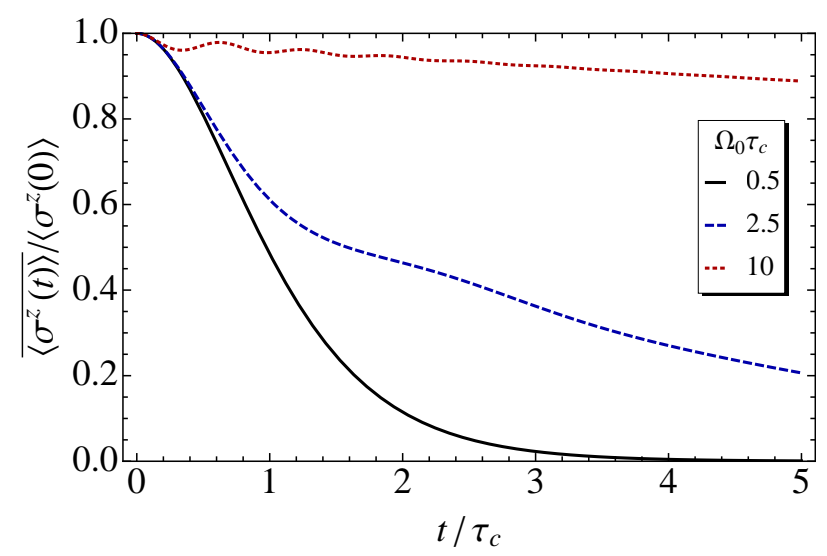

FIG. 2. $\left\langle\overline{S_{z}(t)}\right\rangle$ subject to Gaussian colored noise versus time for nonvanishing constant $\Omega_{0}$ (see legend) and $\omega_{0} \tau_{c}=1$. Equation (32) gives the analytic formula for $\left\langle\overline{S_{z}(t)}\right\rangle$.

of decay given by $\Gamma(t) \approx \omega_{0}^{2} \tau_{c} t$. For times $t \ll \tau_{c}$, the rate of decay is $\Gamma(t) \approx \omega_{0}^{2} t^{2} / 2$. In the absence of an external field, the fluctuations in all three directions remain independent and the average evolution matrix decomposes in the following way:

$$
\left.\overline{\hat{U}(t, 0)}\right|_{\Omega_{0}=0}=\underbrace{\left(\begin{array}{ccc}
e^{-\Gamma(t)} & 0 & 0 \\
0 & e^{-\Gamma(t)} & 0 \\
0 & 0 & 1
\end{array}\right)}_{\text {fluctuations in } z} \underbrace{\left(\begin{array}{ccc}
e^{-\Gamma(t)} & 0 & 0 \\
0 & 1 & 0 \\
0 & 0 & e^{-\Gamma(t)}
\end{array}\right)}_{\text {fluctuations in } y} \underbrace{\left(\begin{array}{ccc}
1 & 0 & 0 \\
0 & e^{-\Gamma(t)} & 0 \\
0 & 0 & e^{-\Gamma(t)}
\end{array}\right)}_{\text {fluctuations in } x} .
$$

Here the first part comes from fluctuations in $z$ and causes the decay of the $x$ and $y$ components of the spin, the second part comes from fluctuations in $y$ and causes the decay of the perpendicular components, and the third part originates from fluctuations in $x$ direction causes the decay of the $y$ and $z$ components of the spin.

Now let us consider finite $\Omega_{0}$. The dependence of $\left\langle\overline{S_{z}(t)}\right\rangle$, the component parallel to the external field $\vec{\Omega}_{0}$, versus time for three values of dimensional parameter $\Omega_{0} \tau_{c}$ is plotted in Fig. 2. As we discussed previously, $z$ component of the average spin decays due to fluctuations in the $x y$ plane, which are affected by the presence of the constant field. For $\Omega_{0} \tau_{c} \ll 1$, the quantity $\left\langle\overline{S_{z}(t)}\right\rangle$ is clearly very similar to the results plotted in Fig. 1 , since $\Gamma_{+}(t) \rightarrow \Gamma(t)$. As $\Omega_{0} \tau_{c}$ increases, the decay of $\left\langle\overline{S_{z}(t)}\right\rangle$ is significantly slowed, and $\Gamma_{+}(t) \rightarrow 0$ in the limit of $\Omega_{0} \tau_{c} \rightarrow \infty$, i.e., the decay is fully suppressed.

Figure 3 plots $\left\langle\overline{S_{x}(t)}\right\rangle$ versus time for the case when $\left\langle S_{y}(0)\right\rangle=0$ and Fig. 4 presents the time dependence of the length of the vector $\left\langle\overline{\vec{S}_{\perp}(t)}\right\rangle=\left(\left\langle\overline{S_{x}(t)}\right\rangle,\left\langle\overline{S_{y}(t)}\right\rangle, 0\right)$. The decay of the components of the average spin perpendicular to 


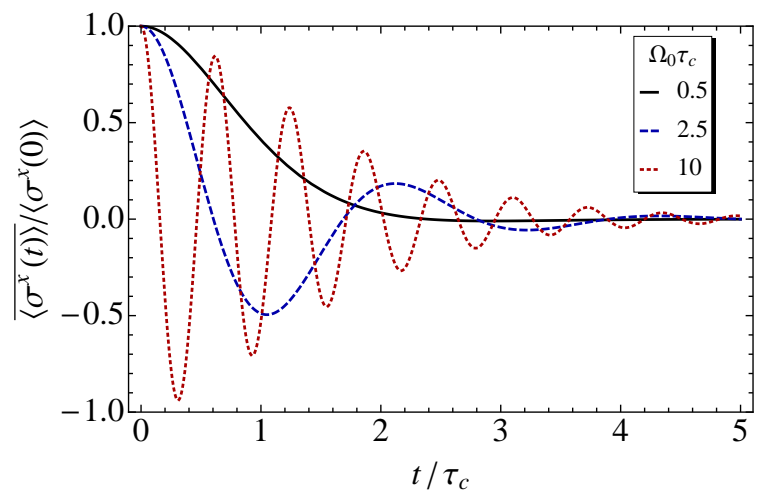

FIG. 3. The average value $\left\langle S_{x}\right\rangle$, when the spin is subject to Gaussian colored noise for nonvanishing constant $\Omega_{0}$ (see legend) and $\omega_{0} \tau_{c}=1$. Equation (30) together with initial condition $\left\langle S_{y}(0)\right\rangle=0$ yield the analytic formula for the function plotted. The average value $\left\langle S_{y}\right\rangle$ for initial condition $\left\langle S_{x}(0)\right\rangle=0$ is identical to the results shown here.

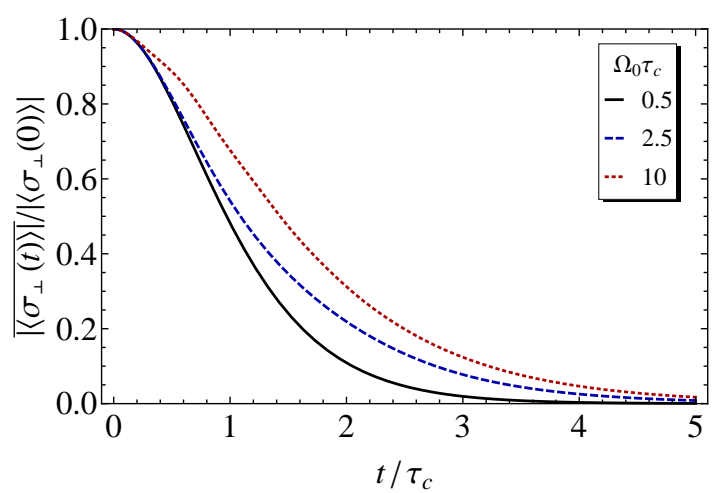

FIG. 4. Average value of $\left|\left\langle\overline{S_{\perp}(t)}\right\rangle\right|=$ $\sqrt{\left\langle\overline{S_{x}(t)}\right\rangle^{2}+\left\langle\overline{S_{y}(t)}\right\rangle^{2}}$ subject to Gaussian colored noise for nonvanishing constant $\Omega_{0}$ (see legend) and $\omega_{0} \tau_{c}=1$. Equations (30) and (31) yield the analytic formula for the function plotted.

the constant field $\vec{\Omega}_{0}$ is not only induced by the fluctuations in the $x y$ plane, but also by those in the $z$ direction [see Eq. [26)]. We have previously seen that the effects of the fluctuations in $x$ and $y$ are inhibited if $\Omega_{0} \tau_{c}$ is large enough. However, the influence of the fluctuations in the direction parallel to applied field is unperturbed by the presence of $\Omega_{0}$. As long as the $z$ component of the field is fluctuating the perpendicular component of the average spin will decay to zero for any value of $\Omega_{0} \tau_{c}$ as $t / \tau_{c} \rightarrow \infty$. For small $\Omega_{0} \tau_{c}$ the decay is, again, similar to the isotropic case shown in Fig. (2), but when $\Omega_{0} \tau_{c}$ increases, the decay is modulated by oscillations which result from the precession of the spin about the external field $\vec{\Omega}_{0}$ with frequency modified by the fluctuations. For $t \gg \tau_{c}$, the oscillation frequency is given by $\Omega_{0}\left(1+\frac{\omega_{0}^{2} \tau_{c}^{2}}{1+\Omega_{0}^{2} \tau_{c}^{2}}\right)$ [see Eq. (28)]. When $\Omega_{0} \tau_{c} \gg 1$ the frequency of precession tends to $\Omega_{0}$ as the frequency modification due to fluctuations, $\Gamma_{-}(t)$ vanishes, in a similar manner as that of $\Gamma_{+}(t)$.

For spin $1 / 2$ system (a TLS), the decay of $\left\langle\overline{S_{x}(t)}\right\rangle$ and $\left\langle\overline{S_{y}(t)}\right\rangle$ correspond to the decay of the coherence of the system, whereas the decay of $\left\langle\overline{S_{z}(t)}\right\rangle$ corresponds to the vanishing of population imbalance. If the constant field is taken along $x$ (as opposed to along $z$ ), decay of $\left\langle\overline{S_{x}(t)}\right\rangle$ is suppressed, and the decoherence time $T_{2} \sim \tau_{c} \Omega_{0}^{2} / \omega_{0}^{2}$, which can be made arbitrarily large (suppressed decay) by increasing $\Omega_{0}$.

The averaged spin vector solution obtained in this section can be extended to the case of a time dependent deterministic field. If $\Omega_{0}$ is a function of time then the frequency of oscillations in the cos and sin functions appearing in the definitions of the decay rates (24) and (25) are to be replaced by an integral, $\Omega_{0} t \rightarrow \int_{0}^{t} \Omega_{0}\left(t^{\prime}\right) d t^{\prime}$.

\section{THE WHITE NOISE LIMIT}

The white noise limit of colored noise involves going to the regime of extremely rapid fluctuations of the field, i.e., vanishing correlation time $\tau_{c}$. Unfortunately, simply taking $\tau_{c} \rightarrow 0$ does not yield the correct limit. If we put $\tau_{c}=0$ in Eq. (29), we obtain that the decay rate of the average spin equals zero, and fluctuations do not affect the evolution of the spin. The proper limit can be obtained if the strength of fluctuations, as given by the variance of the field, $\overline{\omega(t)^{2}}=\omega_{0}^{2}$, is increased as the correlation time $\tau_{c}$ goes to zero, so that $\omega_{0}^{2} \tau_{c}$ remains constant. Thus, we arrive at the limit when the correlation function of the colored noise becomes proportional to a Dirac delta function,

$$
\overline{\omega_{i}(t) \omega_{j}\left(t^{\prime}\right)}=\omega_{0}^{2} e^{-\frac{\left|t-t^{\prime}\right|}{\tau_{c}}} \delta_{i j}=2 \omega_{0}^{2} \tau_{c}\left(\frac{1}{2 \tau_{c}} e^{-\frac{\left|t-t^{\prime}\right|}{\tau_{c}}}\right) \delta_{i j} \frac{\omega_{0}^{2} \tau_{c} \rightarrow D}{\tau_{c} \rightarrow 0} 2 D \delta\left(t-t^{\prime}\right) \delta_{i j}
$$

In the limit of $\kappa(\tau) \rightarrow 2 D \delta(\tau)$ the decay rates $\Gamma_{+}(t)=\Gamma(t)=2 D t$ and $\Gamma_{-}(t)=0$. The decay of the expectation value of the spins are then isotropic and are not affected by the presence of a constant field $\vec{\Omega}_{0}$, i.e.,

$$
\overrightarrow{\langle\vec{S}(t)\rangle}=\left(\begin{array}{ccc}
e^{-\Gamma(t)-\Gamma_{+}(t)} \cos \Gamma_{-}(t) & -e^{-\Gamma(t)-\Gamma_{+}(t)} \sin \Gamma_{-}(t) & 0 \\
e^{-\Gamma(t)-\Gamma_{+}(t)} \sin \Gamma_{-}(t) & e^{-\Gamma(t)-\Gamma_{+}(t)} \cos \Gamma_{-}(t) & 0 \\
0 & 0 & e^{-2 \Gamma_{+}(t)}
\end{array}\right) \hat{R}\left(\Omega_{0} t, z\right)\langle\vec{S}\rangle \rightarrow e^{-4 D t} \hat{R}\left(\Omega_{0} t, z\right)\langle\vec{S}\rangle .
$$


Thus, the spin decay is isotropic, purely exponential with decay rate $4 D$, and is independent of $\Omega_{0}$. Moreover, the precession due to $\vec{\Omega}_{0}$ is unaffected by the fluctuations. In the white noise limit, the precession due to deterministic part of the field and the decoherence due to field fluctuations are completely decoupled.

\section{MEMORY EFFECTS}

In the expression for $\langle\overrightarrow{S(t)}\rangle$ for the case of Gaussian colored noise, the decay rates of the spin depend on the history of the fluctuating field through the integrals of correlation functions. But the decay rate for the white noise case does not depend on history. Based on this observation, we say that spin system has memory if the fluctuating field driving it has finite correlation time. This definition is consistent with a rigorous notion of memory, since $\vec{S}(t)$, understood as a stochastic process, is Markovian [13] (i.e. memoryless) if and only if the noise driving it is white noise. Memory in the system has important consequences, because it allows control of spin decay, through the application of an external field.

The role of memory can be understood as follows. Consider first the case of $\Omega_{0}=0$. Each realization of the fluctuating field $\vec{\omega}(t)$ determines corresponding realization of $\langle\vec{S}(t)\rangle=\hat{U}(t, 0)\langle\vec{S}\rangle$ via the equation of motion, where $\vec{\omega}(t)$ defines an instantaneous rotation axis for the spin vector. Hence, each realization draws a trajectory of the spin on a sphere of radius $|\langle\vec{S}\rangle|$. The evolution can be viewed in a simplified "stroboscopic" picture in which, within a correlation time $\tau_{c}$, the axis $\vec{\omega}(t)$ does not change much, and subsequently, it jumps to a new random value within a range on the order of $\omega_{0}$. Eventually such a sequence of rotations around randomly selected axes "smears" the trajectories of the spin over the whole sphere. This results in the decay of the spin averaged over all realizations.

The situation is different when a constant field along, say, the $z$-axis is present. This becomes clear when we view the evolution in the reference frame rotating with frequency $\Omega_{0}$ around the $z$-axis. Consider a particular realization of the fluctuating field $\vec{\omega}(t)$, and denote it in the rotating frame as $\vec{\omega}^{\prime}(t)$. Since $\omega_{z}^{\prime}(t)=\omega_{z}(t)$, the fluctuations in the direction parallel to the constant field are unchanged when shifted to the rotating frame. On the other hand, the projection of the fluctuating field on the plane perpendicular to $z$-axis, $\vec{\omega}_{\perp}(t)=\left(\omega_{x}(t), \omega_{y}(t), 0\right)$, is modified when viewed in the rotating frame. Within a time of order the correlation time $\tau_{c}$, the projection $\vec{\omega}_{\perp}^{\prime}(t)$ revolves around the $z$-axis with frequency $\Omega_{0}$, and if $\Omega_{0} \tau_{c} \gg 1, \vec{\omega}_{\perp}^{\prime}(t)$ is able to perform many revolutions between jumps. The effect of the jumps becomes negligible in comparison with the systematic rotation. Thus the stochastic character of $\vec{\omega}_{\perp}^{\prime}(t)$ is lost, and the only contribution to the decay caused by fluctuations comes from $\omega_{z}(t)$. A similar effect provides stable motion of a spinning top, and for that reason we will refer to it as a gyroscopic effect. If $\Omega_{0} \tau_{c} \ll 1$, then revolution in between jumps is negligible, so $\vec{\omega}_{\perp}^{\prime}(t) \approx \vec{\omega}_{\perp}(t)$, and by necessity the decay induced by the fluctuations must be almost the same as if there was no constant field at all.

The suppression mechanism of the decay described above is inoperative in the white noise limit where the correlation time vanishes, so the fluctuations of the rotation axis are instantaneous. Hence, the decay caused by white noise cannot be affected by a constant field of finite intensity. Moreover, even if the strength of the fluctuations is small in comparison to $\Omega_{0}$, the effect of fluctuations cannot be neglected if the correlation time is too short.

Note that the important dimensionless parameter here is $\Omega_{0} \tau_{c}$, which can be interpreted as an average angle of precession due to the deterministic field within the time period of $\tau_{c}$. Bearing this in mind, it is clear that the conclusion of this paragraph remains valid even if the field $\Omega_{0}$ varies in time as long as $\tau_{c}\left\langle\Omega_{0}(t)\right\rangle_{\tau_{c}} \equiv \tau_{c}\left(\frac{1}{\tau_{c}} \int_{t}^{t+\tau_{c}} \Omega_{0}\left(t^{\prime}\right) d t^{\prime}\right) \gg 1$. Moreover, the reasoning used above shows that the same decay suppression effects apply even for non-Gaussian noise as long as it has non-vanishing correlation time. We verify this assessment in the following section.

\section{NON-GAUSSIAN PROCESS: TELEGRAPH NOISE}

In this section we present an example of a non-Gaussian process, telegraph noise, i.e., dichotomic noise corresponding to a memoryless continuous-time stochastic process that jumps between two distinct values, sometimes called burst noise [13, 27]. We consider telegraph noise in one component of the field, $\omega_{z}(t)$. The Rabi frequency of the fluctuating field, $\omega_{z}(t)$, jumps randomly between $+\omega_{0}$ and $-\omega_{0}$, with a jump rate $w=\left(2 \tau_{c}\right)^{-1}$. The average value and the correlation function of such a process is given by

$$
\overline{\omega_{z}(t)}=0, \quad \overline{\omega_{z}(t) \omega_{z}\left(t^{\prime}\right)}=\omega_{0}^{2} e^{-2 w\left|t-t^{\prime}\right|}=\omega_{0}^{2} e^{-\frac{\left|t-t^{\prime}\right|}{\tau_{c}}} .
$$

Because telegraph noise is non-Gaussian, the correlation function $\kappa\left(t-t^{\prime}\right)=\omega_{0}^{2} e^{-\frac{\left|t-t^{\prime}\right|}{\tau_{c}}}$ does not contain all the information regarding the process. 
Telegraph noise can model the effect of a spin $1 / 2$ impurity atom near the spin [2, 3, [5]. The impurity spin has probabilities $p_{+}$and $p_{-}$for being in the spin-up and spin-down state, and a hopping rate for transferring from one spin state to the other. Neglecting the back-action of the magnetic moment on the impurity, this model reduces to telegraph noise.

Our goal is to find the average evolution matrix $\overline{\hat{U}(t, 0)}$ for the telegraph noise case. The technique we have used previously to find the average does not work in the case of telegraph noise, because unlike with Gaussian processes, the closed form of the cumulant generating functional is not known. Fortunately, it is still possible to find an exact, analytical formula for $\overline{\hat{U}(t, 0)}$ if the fluctuations are parallel to the constant field. One way of solving for $\overline{\hat{U}(t, 0)}$ is to follow Ref. [28]. We can write the average evolution operator as $\overline{\hat{U}(t, 0)}=\overline{\hat{U}(t, 0)}+\overline{\hat{U}(t, 0)}_{-}$, where $\overline{\hat{U}(t, 0)}_{+}$are the averages conditional on $\omega_{z}(t)$ being equal to $\pm \omega_{0}$. These averages vary in time because of equation of motion (77), and because $\omega_{z}(t)$ jumps. Thus,

$$
\begin{aligned}
& \frac{d}{d t}{\overline{\hat{U}(t, 0)_{+}}}_{+}=\left(\Omega_{0}+\omega_{0}\right) \hat{\varepsilon}^{z}{\overline{\hat{U}(t, 0)_{+}}}_{+}-\frac{1}{2 \tau_{c}}{\overline{\hat{U}(t, 0)_{+}}}_{+}+\frac{1}{2 \tau_{c}} \overline{\hat{U}(t, 0)}_{-}, \\
& \frac{d}{d t}{\overline{\hat{U}(t, 0)_{-}}}=\left(\Omega_{0}-\omega_{0}\right) \hat{\varepsilon}^{z}{\overline{\hat{U}(t, 0)_{-}}}_{-}-\frac{1}{2 \tau_{c}} \overline{\hat{U}(t, 0)}_{-}+\frac{1}{2 \tau_{c}}{\overline{\hat{U}}(t, 0)_{+}} .
\end{aligned}
$$

Adding and subtracting these equations, we obtain

$$
\begin{aligned}
\frac{d}{d t} \overline{\hat{U}(t, 0)} & =\Omega_{0} \hat{\varepsilon}^{z} \overline{\hat{U}(t, 0)}+\omega_{0} \hat{\varepsilon}^{z} \overline{\Delta \hat{U}(t, 0)} \\
\frac{d}{d t} \overline{\Delta \hat{U}(t, 0)} & =\Omega_{0} \hat{\varepsilon}^{z} \overline{\Delta \hat{U}(t, 0)}+\omega_{0} \hat{\varepsilon}^{z} \overline{\hat{U}(t, 0)}-\frac{1}{\tau_{c}} \overline{\Delta \hat{U}(t, 0)},
\end{aligned}
$$

where $\overline{\Delta \hat{U}(t, 0)}=\overline{\hat{U}(t, 0)}_{+}-\overline{\hat{U}(t, 0)}_{-}$. The next step is to eliminate $\overline{\Delta \hat{U}(t, 0)}$ from Eq. (40) by substituting the solution to Eq. (41), with the initial condition $\overline{\hat{U}(0,0)}_{ \pm}=\hat{1} / 2$. This yields a closed equation for average evolution matrix:

$$
\frac{d}{d t} \overline{\hat{U}(t, 0)}=\Omega_{0} \hat{\varepsilon}^{z} \overline{\hat{U}(t, 0)}+\omega_{0}^{2} \int_{0}^{t} d t^{\prime} e^{-t^{\prime} / \tau_{c}}\left(\hat{\varepsilon}^{z}\right)^{2} \overline{\hat{U}\left(t-t^{\prime}, 0\right)} .
$$

Note the particular form of the "memory integral" in the equation (42), where the dynamical variable is evaluated at times previous to $t$. In this case the system clearly has memory and it remembers not only the history of the noise but also its own history. It is easy to check that the solution to this equation that satisfies the initial condition $\overline{\hat{U}(0,0)}=\hat{1}$ is given by

$$
\overline{\hat{U}(t, 0)}=e^{-t / 2 \tau_{c}}\left[\cosh \left(\hat{\gamma} \frac{t}{2 \tau_{c}}\right)+\hat{\gamma}^{-1} \sinh \left(\hat{\gamma} \frac{t}{2 \tau_{c}}\right)\right] \hat{R}\left(\Omega_{0} t, z\right)
$$

where

$$
\hat{\gamma}=\left(\begin{array}{ccc}
\sqrt{1-4 \omega_{0}^{2} \tau_{c}^{2}} & 0 & 0 \\
0 & \sqrt{1-4 \omega_{0}^{2} \tau_{c}^{2}} & 0 \\
0 & 0 & 1
\end{array}\right)
$$

The average expectation values of the spin are obtained by applying the average evolution matrix (43) to the initial vector of spin operators, $\langle\overline{\langle\vec{S}(t)}\rangle=\overline{\hat{U}(t, 0)}\langle\vec{S}(0)\rangle$, and this yields,

$$
\begin{aligned}
& \left\langle\overline{S_{x}(t)}\right\rangle=e^{-\frac{t}{2 \tau_{c}}}\left[\cosh \left(\sqrt{1-4 \omega_{0}^{2} \tau_{c}^{2}} \frac{t}{2 \tau_{c}}\right)+\frac{\sinh \left(\sqrt{1-4 \omega_{0}^{2} \tau_{c}^{2}} \frac{t}{2 \tau_{c}}\right)}{\sqrt{1-4 \omega_{0}^{2} \tau_{c}^{2}}}\right]\left[\cos \left(\Omega_{0} t\right)\left\langle S_{x}\right\rangle-\sin \left(\Omega_{0} t\right)\left\langle S_{y}\right\rangle\right], \\
& \left\langle\overline{S_{y}(t)}\right\rangle=e^{-\frac{t}{2 \tau_{c}}}\left[\cosh \left(\sqrt{1-4 \omega_{0}^{2} \tau_{c}^{2}} \frac{t}{2 \tau_{c}}\right)+\frac{\sinh \left(\sqrt{1-4 \omega_{0}^{2} \tau_{c}^{2}} \frac{t}{2 \tau_{c}}\right)}{\sqrt{1-4 \omega_{0}^{2} \tau_{c}^{2}}}\right]\left[\cos \left(\Omega_{0} t\right)\left\langle S_{y}\right\rangle+\sin \left(\Omega_{0} t\right)\left\langle S_{x}\right\rangle\right], \\
& \left\langle\overline{S_{z}(t)}\right\rangle=\left\langle S_{z}\right\rangle .
\end{aligned}
$$

Neither constant field $\Omega_{0}$, nor field fluctuations $\omega_{z}(t)$ couple to the $z$ component of the spin, hence it remains constant throughout the evolution. The effects of $\Omega_{0}$ and $\omega_{z}(t)$ on the components of the spin in $x y$ plane are simply superposed: 
constant field causes trivial precession around $z$ axis, while the noise makes the $x$ and $y$ spin components decay on average. Moreover, if the constant field and the fluctuating field are parallel, the gyroscopic effect can not be observed. The only memory effect present in this case is a quadratic behavior in $t$ of the decay rate at $t \ll \tau_{c}$. Figure 5 plots $\left\langle\overline{S_{x}(t)}\right\rangle$ versus time for telegraph noise in $\omega_{z}(t)$ for the case of no static external magnetic present. As $\omega_{0} \tau_{c}$ increases, $\left\langle\overline{S_{x}(t)}\right\rangle$ decays more quickly, but for $\omega_{0} \tau_{c}>1 / 2$, the square root $\sqrt{1-4 \omega_{0}^{2} \tau_{c}^{2}}$ becomes imaginary and the solution changes form from pure decay to decay with oscillations.

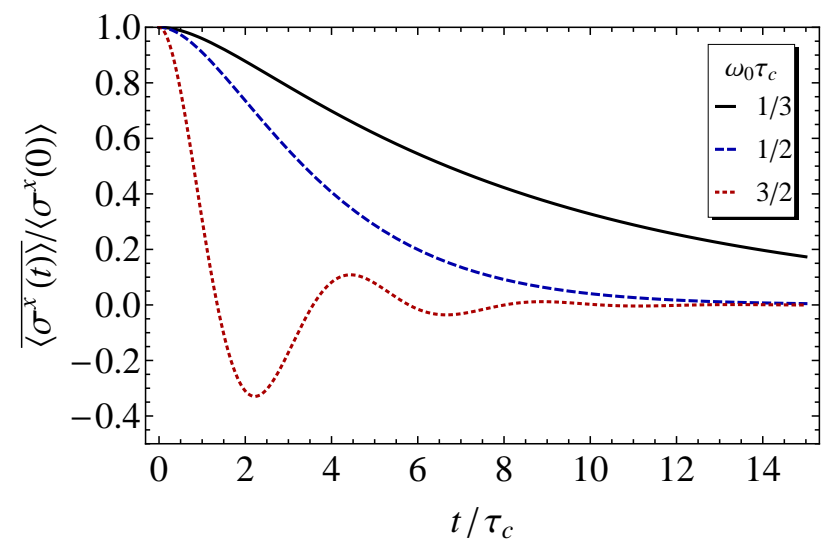

FIG. 5. $\left\langle\overline{S_{x}(t)}\right\rangle$ versus time for a spin subject to telegraph noise in only the $z$-component of the field, $\omega_{z}(t)$, and $\Omega_{0}=0 .\left\langle\overline{S_{y}(t)}\right\rangle$ is identical to $\left\langle\overline{S_{x}(t)}\right\rangle$. Nonvanishing values of $\Omega_{0}$ simply cause precession of the spin about the $z$ axis.

It is of interest to see what happens when noise is present in the component of the field perpendicular to the constant field $\Omega_{0}$, e.g., in $\omega_{x}(t)$. Unfortunately, in this case, it is not possible to obtain a closed form expression for the average evolution matrix. According to the previous discussion, one would expect that, since telegraph noise is colored noise (the correlation time $\tau_{c}$ is finite), the gyroscopic effect should work in a fashion similar to the Gaussian colored noise example. This is indeed the case. Numerical solutions of $\left\langle\overline{S_{z}(t)}\right\rangle$ versus time are plotted in Fig. 6 and suppression of the decay is more efficient with increasing $\Omega_{0} \tau_{c}$.

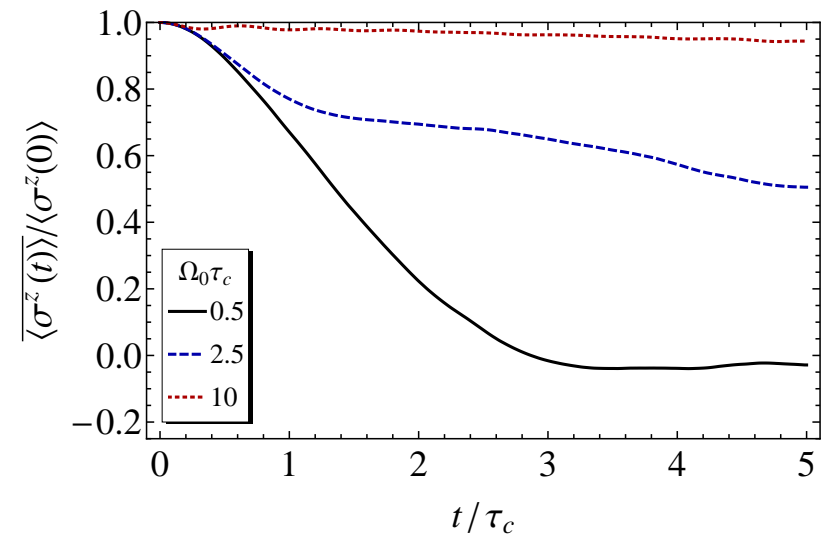

FIG. 6. $\left\langle\overline{S_{z}(t)}\right\rangle$ versus time for a spin subject to telegraph noise only in the $x$ direction, with $\omega_{0} \tau_{c}=1$. The deterministic magnetic field with Rabi frequency $\Omega_{0}$ is taken to be in the $z$ direction, so it is perpendicular to the noise. As the parameter $\Omega_{0} \tau_{c}$ is increased (see legend), suppression of the decay of the spin increases.

\section{SUMMARY AND CONCLUSION}

We introduced a stochastic model for spin in the presence of a deterministic magnetic field and a fluctuating magnetic field (noise), and we derived stochastic equations of motion for the spin vector $\langle\vec{S}(t)\rangle$. The environmental degrees of freedom, represented by the fluctuations of the field, are eliminated by taking the average over the fluctuations to 
obtain $\langle\overrightarrow{\vec{S}(t)}\rangle$. We made the external noise assumption, wherein no back-action of the system on the environment is present. The most pronounced consequence of this assumption is that the system does not go into equilibrium with a thermal environment, but instead goes to the most democratic density matrix, i.e., the system tends towards the completely mixed state, and $\langle\overrightarrow{S(t)}\rangle \rightarrow 0$ as $t \rightarrow \infty$. This is in contradistinction to the case of noise experienced by a system in contact with a bath wherein the bath affects the system and vice versa. The lack of mutual interaction (back-action) means that the fluctuation-dissipation theorem [29] cannot be applied, and a thermal equilibrium state of the system is not obtained at large times. If back-action on the bath due to the system is present, the source of the fluctuations and the dissipation is the same, they are connected through fluctuation-dissipation theorem, and thermal equilibrium of the system must result.

We explicitly considered Gaussian colored noise, Gaussian white noise and non-Guassian telegraph noise. From our studies we conclude that if the system has memory, i.e., it has a finite correlation time, then the system can be manipulated by means of an external magnetic field, and the decoherence induced by the fluctuations can be significantly suppressed. However, in the white noise limit, when the correlation function tends to a Dirac delta function, the decoherence cannot be controlled in this way. We introduced simple and intuitive considerations for why the suppression of decay can be achieved for any stochastic process as long as the correlation time is long enough.

\section{ACKNOWLEDGMENTS}

This work was supported in part by grants from the Israel Science Foundation (No. 2011295), and the James

Franck German-Israel Binational Program. P.S. acknowledges the Foundation for Polish Science International PhD Projects Programme co-financed by the EU European Regional Development Fund, and the National Science Centre DEC-2011/03/D/ST2/00200. M.T. acknowledges financial support of the National Science Centre. We are grateful Doron Cohen and Marek Kuś for useful conversations related to this work.

[1] R. P. Feynman and F. L. Vernon, Ann. Phys. (N.Y.) 24, 118 (1963).

[2] T. Itakura and Y. Tokura, Phys. Rev. B67, 195320 (2003).

[3] B. Cheng, Q. H. Wang, and R. Joynt, Phys. Rev. A78, 022313 (2008).

[4] J. Bergli, Y. M. Galperin, and B. L. Altshuler, New J. Phys. 11, 025002 (2009).

[5] A. Aharony, S. Gurvitz, O. Entin-Wohlman and S. Dattagupta, Phys. Rev. B82, 245417 (2010).

[6] C. Bennett, Physics Today 48 (10), 24 (1995); D. P. DiVincenzo, Science 270, 255 (1995); A. M. Steane, Rep. Prog. Phys. 61, 117 (1998).

[7] D. Budker and M. Romalis, Nature Phys. 3, 227 (2007).

[8] J. Vanier and C. Audoin, The Quantum Physics of Atomic Frequency Standards, (Adam Hilger IOP Publishing Ltd., Bristol, 1989); P. Lemonde, et al., "Cold-Atom Clocks on Earth and in Space", in Frequency Measurement and Control, (Springer-Verlag, Berlin, 2001); Topics Appl. Phys. 79, 131-153 (2001).

[9] D. Loss and D. P. DiVincenzo, Phys. Rev. A57, 120 (1998); T. Tanamoto, ibid. 61, 022305 (2000).

[10] J.M. Taylor et al., Phys. Rev. B 76, 035315 (2007).

[11] L. Childress et al., Science 314, 281 (2006); R. Hanson et al., Science 320, 352 (2008); J. R. Maze et al., Nature 455, 644 (2008).

[12] A. Bermudez, F. Jelezko, M. B. Plenio, and A. Retzker, Phys. Rev. Lett. 107, 150503 (2011).

[13] N. G. Van Kampen, Stochastic Processes in Physics and Chemistry, (Elsevier Science, 1997).

[14] P. E. Kloeden and E. Platen, Numerical Solution of Stochastic Differential Equations, (Springer, 2011).

[15] M. C. Valsakumar, J. of Stat. Phys. 39, 347 (1985).

[16] N. G. van Kampen, J. of Stat. Phys. 54, 1289 (1989).

[17] N. Bar-Gill, L. M. Pham, C. Belthangady, D. Le Sage, P. Cappellaro, J. R. Maze, M. D. Lukin, A. Yacoby, and R. Walsworth, Nat. Commun. 3, 858 (2012).

[18] P. W. Anderson and P. R. Weiss Rev. Mod. Phys 25, 269 (1953)

[19] J. R. Klauder and P. W. Anderson, Phys. Rev. 125, 912 (1962).

[20] G. de Lange et al., Science 330, 60 (2010).

[21] V.V. Dobrovitski, A.E. Feiguin, R. Hanson, D.D. Awschalom, Phys. Rev. Lett. 102, 237601 (2009).

[22] V. Dikovsky, Y. Japha, C. Henkel and R. Folman, Eur. Phys. J. D. 35, 87 (2005)

[23] The number of independent parameters needed to characterize the density matrix of a spin $S$ system is $(2 S+1)^{2}-1$, so for $S=1 / 2$ the three quantities $\left\langle S_{i}\right\rangle$ are sufficient, but for higher $S$, the number of parameters exceeds the number of expectation values of the spin components.

[24] J. L. Doob, Ann. Math. Statist. 15, 229 (1944).

[25] N. G. van Kampen, Physica 74, 239 (1974).

[26] R. Kubo, J. Phys. Jap. 17, 1100 (1962). 
[27] C. W. Gardiner, Handbook of Stochastic Methods, (Springer, 2004).

[28] N. G. van Kampen Physica 70, 222 (1973).

[29] H. B. Callen and T. A. Welton, Phys. Rev. 83, 34 (1951). 